\title{
Ion temperature gradient turbulence modification in quasi-axisymmetry
}

\author{
Samuel A. Lazerson,,${ }^{1, *}$ Pavlos Xanthopoulos, ${ }^{2}$ Harry Mynick,,${ }^{1}$ and David Gates ${ }^{1}$ \\ ${ }^{1}$ Princeton Plasma Physics Laboratory \\ ${ }^{2}$ Max-Planck Intitut-für-Plamaphysik
}

(Dated: December 4, 2018)

\begin{abstract}
The large flexibility of the proposed QUASAR facility [Gates et al. 2017] is leveraged in order to explore the effect of magnetic shear on electrostatic ITG turbulence. The QUASAR facility is a reimagining of the NCSX experiment utilizing and expanding upon the already constructed coil set. Recent work using fixed boundary optimization of the LI383 equilibrium (upon which QUASAR is based) has suggested possible improvements to ITG turbulence [Mynick et al. 2014]. In this work, a different approach is taken wherein a series of self-consistent free boundary VMEC equilibria are developed for QUASAR. These equilibria assume temperature and density profiles consistent with $2 \%$ beta and ohmic current drive. In each configuration the toroidal field coils are energized to different values and the STELLOPT code is used to vary the modular coil current and net toroidal current. The edge value of rotational transform is targeted in the optimization, producing a magnetic shear scan. These configurations all share similar neoclassical transport levels while non-linear GENE flux tube simulations show up to a factor of four change in electrostatic ITG turbulence at various radii. Comparisons to proxy functions and linear flux tube runs are also made. This work highlights the capability of the QUASAR experiment as a tool to explore transport in 3D magnetic fields and the possibility of the further improvements to stellarators through optimization.
\end{abstract}

\footnotetext{
*lazerson@pppl.gov
} 


\section{INTRODUCTION}

The numerical optimization of stellarator configurations for improved transport properties [1] has yielded devices with tokamak levels of neoclassical transport [2-4] and suggests that devices with reduced turbulent transport may be developed as well [5]. Stellarators have been shown to benefit from three-dimensional effects on a flux surface, causing a reduction of turbulence levels [6]. The QUASAR stellarator provides a setting in which electrostatic Ion Temperature Gradient (ITG) turbulence may be explored [7, 8]. In this work, a set of five experimentally realizable equilibria are developed which scan magnetic shear $(d \iota / d \rho)$ at fixed edge rotational transform $(\iota)$. This produces configurations which vary from traditional lowshear stellarator configurations to ones with a 'tokamak-like' rotational transform profiles. The resulting variation in ITG turbulence properties are investigated with linear and nonlinear gyrokinetic calculations, suggesting new modifications to existing ITG turbulence proxy functions.

The QUASAR stellarator is a re-envisioning of the cancelled National Compact Stellarator Experiment (NCSX) [9-11]. In QUASAR, the NCSX coil set and vacuum vessel is reused with the possibility of additional coils. For this work the auxiliary ohmic and vertical field coil sets, as envisioned for NCSX, are utilized. It should be noted that the optimized modular stellarator coils and toroidal field coils have already been manufactured. Thus, the QUASAR facility is a quasi-axisymmetric stellarator capable of Ohmic current drive and heating. The device is envisioned to be run with stable discharge conditions for 3 to 5 seconds.

This study showcases the unique capabilities of QUASAR, provides an experimental path towards validating turbulence predictions in stellarators, and hints at the possibility of a fully transport optimized stellarator reactor. Unlike modern high performance stellarators (LHD and W7-X) the QUASAR stellarator has a large configuration space [12]. This is in part due to its extensive coil set which includes three optimized stellarator coils, toroidal field coils, poloidal field coils, and ohmic coil. The ability to drive Ohmic current is shared by only one other stellarator in the world, the Compact Toroidal Hybrid [13]. The low levels of predicted neoclassical transport allow QUASAR to serve as a stellarator turbulence teststand. Moreover, the quasi-axisymmetric nature of the device should allow comparison with existing tokamak turbulence studies [14]. Finally, if predictions of a turbulence optimized 
stellarator can be validated in experiment, the stellarator reactor design may become preferable to tokamak configurations. In the next section we discuss the tools and methods used in this work. In section III, we present the results of this analysis followed by a discussion of the results in section IV.

\section{METHOD}

An exploration of ITG turbulence in the QUASAR device is conducted using the VMEC [15], STELLOPT [16, 17], and GENE [18, 19] codes. The VMEC code is used to solve for free-boundary ideal MHD equilibria with continuously nested flux surfaces in the QUASAR stellarator. Care is taken to tailor profiles which are consistent with a $\beta \sim 2 \%$ ohmic discharge. The STELLOPT code is used to optimize the equilibrium inputs (coil currents, current, and enclosed flux) so that ITG turbulence can be evaluated through a shear scan in the device. Edge rotational transform and plasma volume serve as targets. The resulting equilibria are then evaluated at multiple radial locations to provides estimates of ITG driven heat flux. Such an analysis highlights the unique capabilities of the QUASAR facility to explore turbulence and its optimization.

The VMEC code is used in this work to calculate the free boundary three dimensional ideal MHD equilibrium of the QUASAR device. This is achieved by minimizing the ideal MHD energy functional

$$
W=\int\left(\frac{p(\psi)}{\gamma-1}+\frac{B^{2}}{2 \mu_{0}}\right) d V
$$

subject to the the nested flux surface constraint $\vec{B} \cdot \nabla \psi=0$, where $p$ is the plasma pressure, $\gamma$ the adiabatic index, $\vec{B}$ the magnetic field, $\psi$ the toroidal flux, $\mu_{0}$ the permittivity of free space, and integration is over the domain of the plasma equilibrium. Free boundary simulations are made possible through consideration of the vacuum field energy on the plasma boundary [20]. Assuming temperature profiles of the form $T_{k}(s)=T_{k 0}\left(1-s^{2}\right)$ (where $s=\psi / \psi_{\text {edge }}$ is the normalized toroidal flux and index $k=i, e$ denotes the ion and electron species), a $\beta=2 \%$ equilibrium can be constructed. In this scenario $T_{i 0}=3.5 \mathrm{keV}$, $T_{e 0}=1.5 \mathrm{keV}$, and the electron density is assumed constant at $5 \times 10^{19} \mathrm{~m}^{-3}$. These profiles are chosen to mimic an ohmic discharge in the experiment. As a result, the current density profile has a form $I^{\prime}(s)=\left(1-s^{2}\right)$ consistent with the electron temperature. All profiles are specified using the 100 point Akima spline parameterization [21]. The net toroidal current 
TABLE I: Target parameters used in the development of the five equilibrium scenarios. Weighting $1 / \sigma$ chosen to focus on edge rotational transform while preserving plasma shape.

\begin{tabular}{c|cc} 
Target Parameter & Value Weight \\
\hline Edge Iota & 0.616 & 100 \\
Aspect Ratio & 4.44 & 1.0 \\
$R B_{\text {Toroidal }}$ & 2.35 & 1.0 \\
Volume & 3.06 & 1.0
\end{tabular}

is left as a free parameter allowing edge iota to be held fixed while the magnetic shear is varied.

A series of ideal equilibria which scan magnetic shear at fixed edge rotational transform are generated using the STELLOPT code. The modified Levenberg-Marquardt algorithm $[22,23]$ is used to target an edge rotational transform value of $\iota=0.616$ for five equilibria at different levels of auxiliary toroidal field coil current. In order to simplify the optimization, the three modular coil types of the stellarator coil-set are treated as one coil system, holding their relative strengths fixed. Table I depicts the target parameters used in the optimization and their relative weightings. In section III the resulting five equilibria are described.

The end goal of this work is to demonstrate that the QUASAR facility can be used to test electrostatic ITG turbulence. This is done through evaluation of these equilibria using the gyrokinetic code GENE. Recently the STELLOPT code has been interfaced to the GIST code [24] allowing it to directly calculate the geometric quantities necessary for linear and non-linear runs of GENE. A comparison between the standalone GIST computation and that incorporated into STELLOPT can be seen in figure 1. This has allowed the development of ITG and TEM proxy functions for turbulence $[5,25]$ and the inclusion of linear GENE calculations directly in the optimizer. This last feature has been utilized in this work to simplify calculations of the radial profiles of linear growth rates. Standalone non-linear flux tube runs are performed for select flux tubes. Such simulations are still too computationally expensive to directly incorporate in the optimizer. 

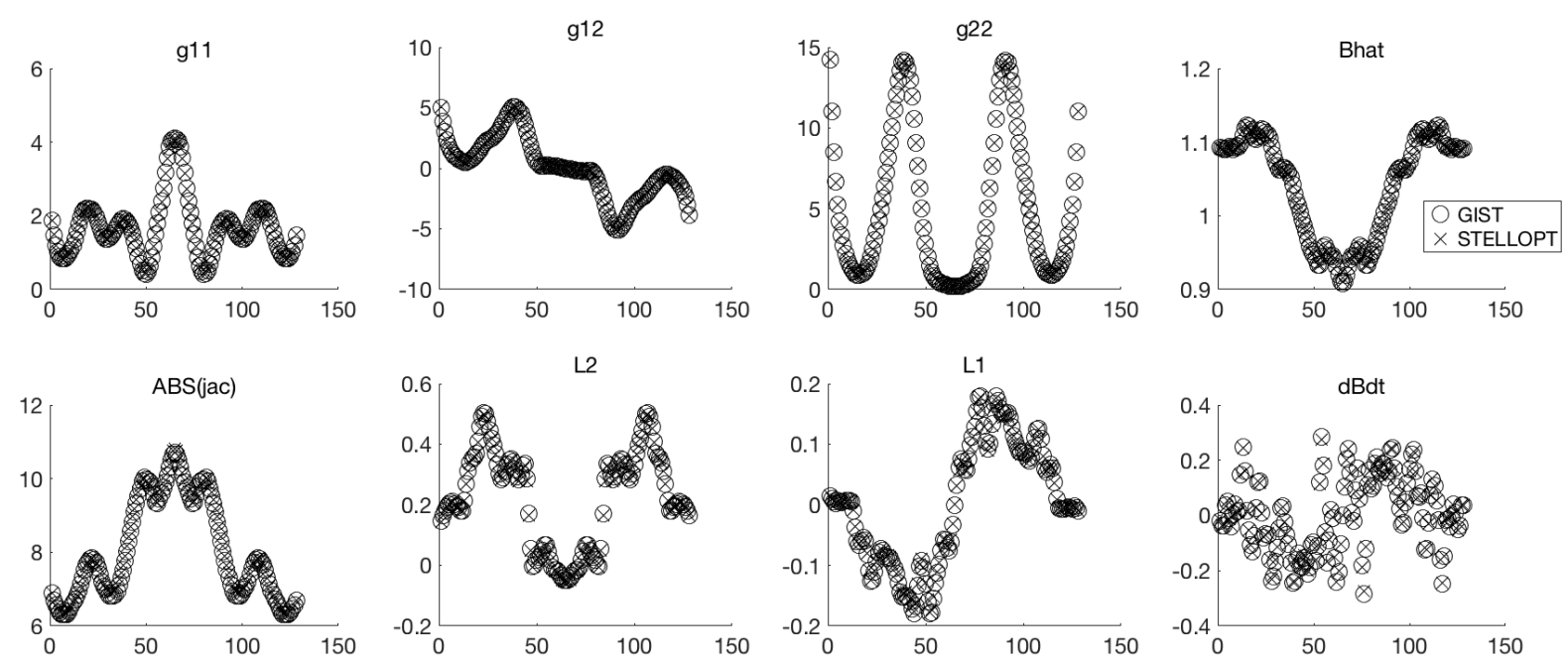

FIG. 1: Comparison between flux tube geometry parameters as calculated by GIST and the implementation of GIST in STELLOPT, showing agreement to within a percent for all quantities. The LI383 equilibrium is used to generate these runs where the flux tube is taken at $\rho \sim 0.7$ (normalized) which passes through the outboard midplane (point of maximum radial curvature). $\mathrm{X}$-axes labels grid points along flux the flux tube for a single poloidal transit and y-axes are taken to be in arbitrary units.

\section{RESULTS}

In this work, five ohmic discharge type scenarios, varying shear but holding edge rotational transform fixed, are developed using the STELLOPT code. These scenarios are then evaluated for their electrostatic ITG turbulence properties using proxy functions, linear growth rates, and full non-linear flux tube simulations. The variation of the non-linear turbulent heat flux is found to exceed factors of two at multiple radii in the plasma.

The STELLOPT optimizer was used to develop the five scenarios based on a variation of the axisymmetric toroidal field generated by the auxiliary toroidal field coils of the QUASAR device. Table II lists the free parameters for the optimization and the optimized values. These values all appear reasonable for the QUASAR experiment. Figure 2 depicts the evolution of $\chi^{2}$ during the optimization for the $100 k A$ case. The Levenberg method clearly searches along two paths during the first iteration (initial equilibrium denoted as 1). The two paths equate to Gauss-Newton trajectory and the gradient descent. The descent is dominated by the edge rotational transform constraint, although all targeted quantities are 
TABLE II: Toroidal field (TF) coil currents, modular coil (MC) current scaling factor, equilibrium enclosed toroidal flux, and toroidal currents which define each of our five scenarios investigated.

\begin{tabular}{c|ccc} 
TF Coil Current & MC Scale Factor Enclosed Flux Toroidal Current \\
\hline $100 \mathrm{kA}$ & 0.85 & $0.511 \mathrm{~Wb}$ & $-176 \mathrm{kA}$ \\
$50 \mathrm{kA}$ & 0.93 & $0.509 \mathrm{~Wb}$ & $-135 \mathrm{kA}$ \\
$0 \mathrm{kA}$ & 1.00 & $0.506 \mathrm{~Wb}$ & $-85.7 \mathrm{kA}$ \\
$-50 \mathrm{kA}$ & 1.07 & $0.505 \mathrm{~Wb}$ & $-27.8 \mathrm{kA}$ \\
$-100 \mathrm{kA}$ & 1.08 & $0.504 \mathrm{~Wb}$ & $44.6 \mathrm{kA}$
\end{tabular}

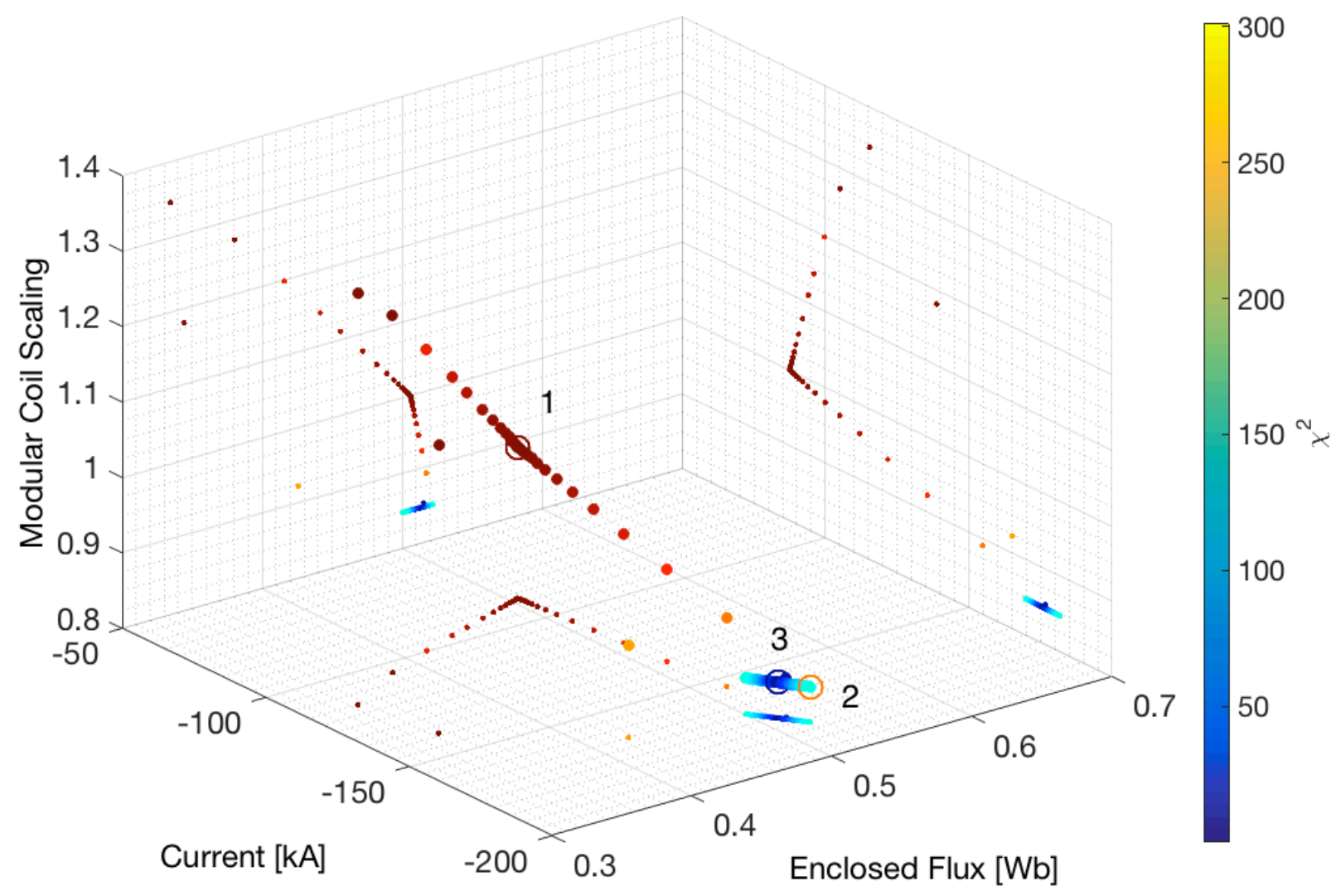

FIG. 2: Depiction of the minimization performed by STELLOPT in the three dimensional parameter space. Successive minima are denoted by circles and numbering, while the data points are projected along the walls of the plot. The $100 \mathrm{kA}$ configuration is shown. 


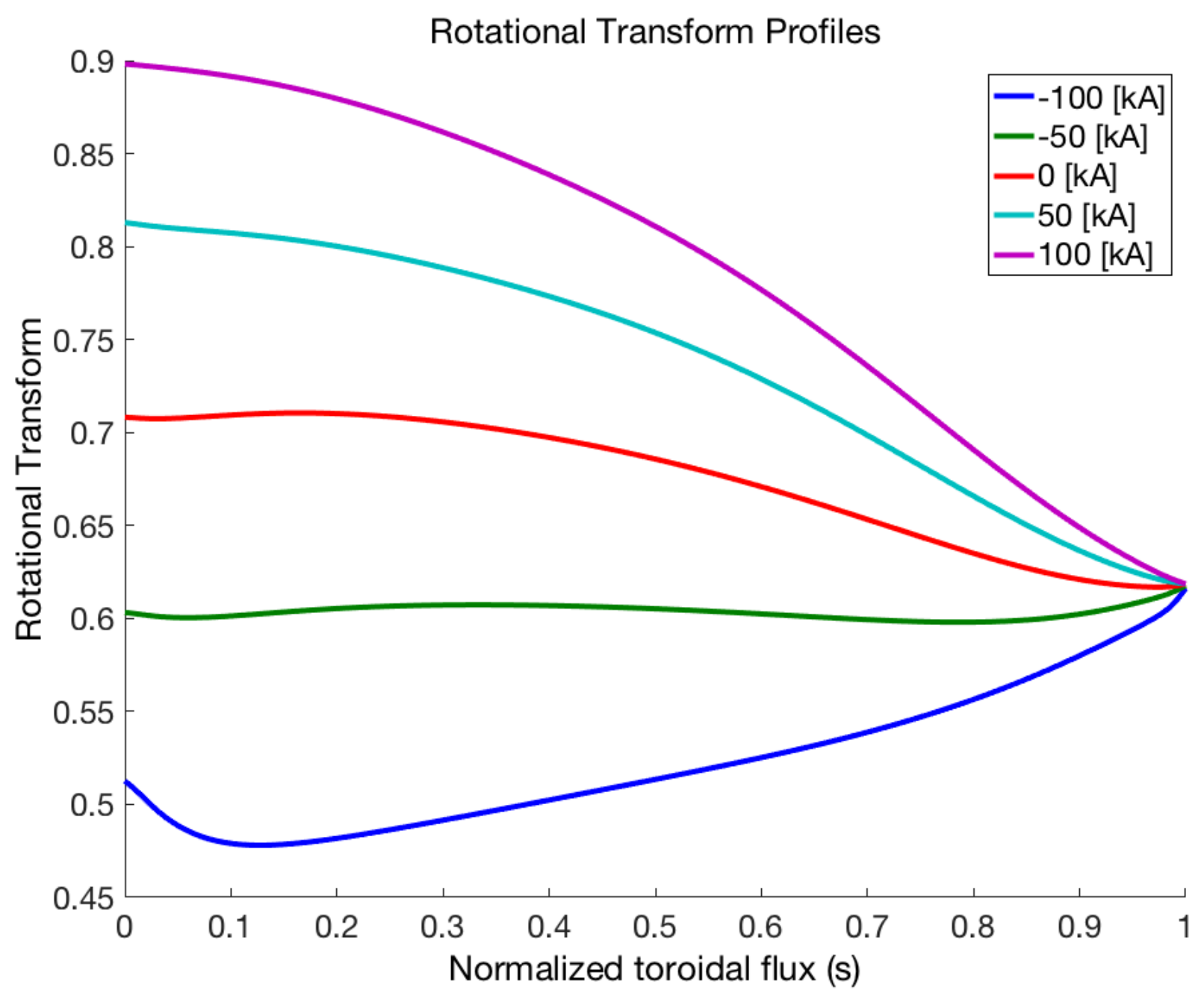

FIG. 3: Rotational transform profiles for the five QUASAR equilibria. The cases scan from stellarator like $(-100 k A)$ to tokamak-like $(100 k A)$ rotational transform profiles.

minimized. This is because the contributions to $\chi^{2}$ are fairly orthogonal with respect to their dependence on the varied parameters.

This rather simple optimization task resulted in five unique equilibria for the QUASAR device. Figure 3 depicts rotational transform profiles for these five equilibria. The magnetic shear has clearly been varied while holding the edge rotational transform fixed. While one may consider the case with zero auxiliary toroidal field $(0 \mathrm{kA})$ to be the 'pure stellarator case,' the rotational transform suggests a different labeling of the equilibria. Specifically, the configuration where the applied toroidal field opposes that generated by the modular coils $(-100 k A)$ has a more stellarator-like profile. Thus it will be referred to as the 'stellaratorlike' case for this work. The configuration in which the applied toroidal field is in the 


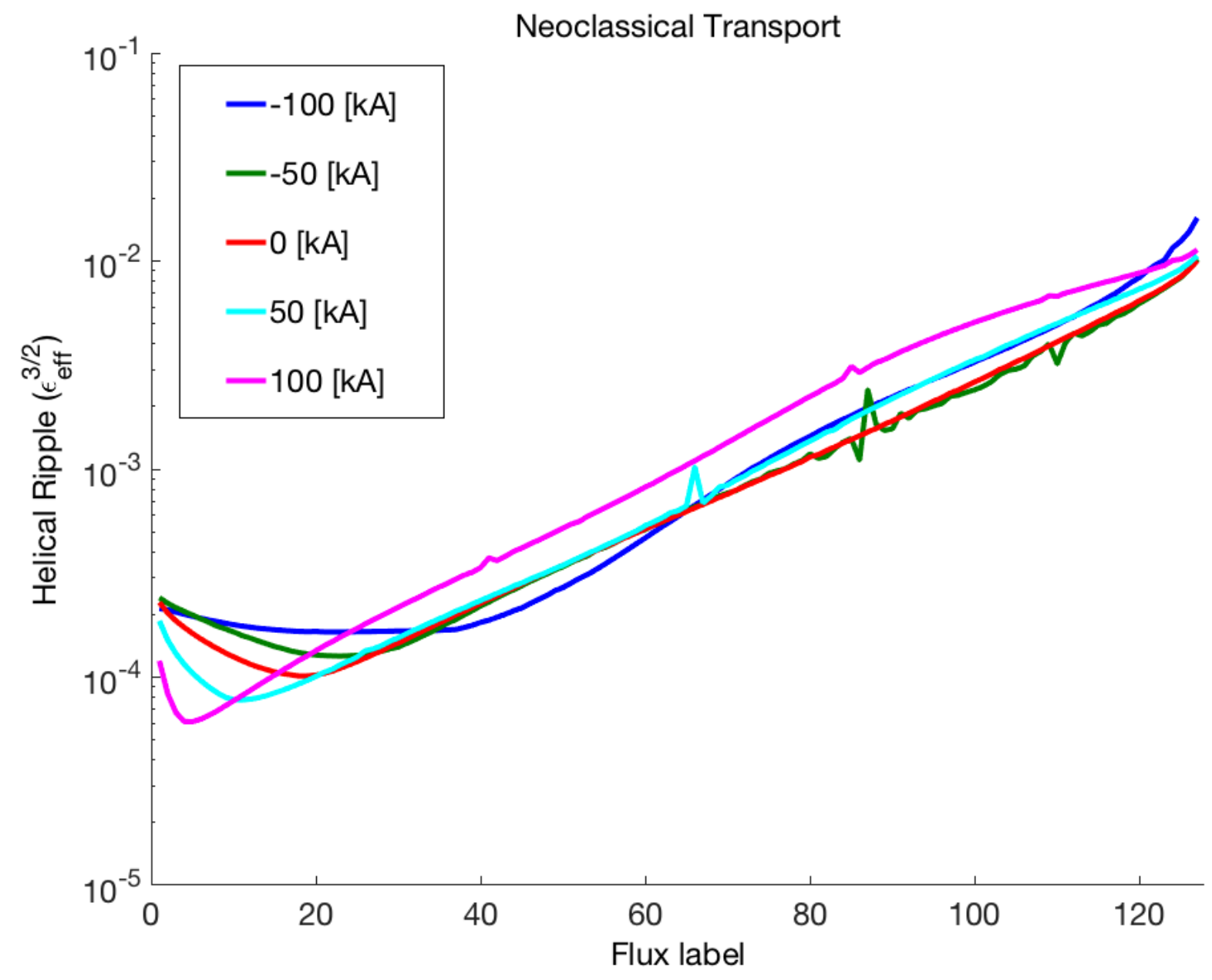

FIG. 4: Helical ripple profiles for the five QUASAR equilibria, suggesting minimal variation in neoclassical transport between configurations. The low values also suggest transport would be dominated by turbulent heat fluxes.

direction of that generated by the modular coil has a more tokamak-like profile. Thus it will be referred to as the 'tokamak-like' case for this work $(100 \mathrm{kA})$.

Examination of the five configurations in more detail highlights some interesting features. The neoclassical transport (figure 4 ), as characterized by the helical ripple parameter $\left(\epsilon_{\text {eft }}^{3 / 2}\right)$, is low in all five configurations [26]. This suggests that transport for these configurations will be dominated by anomalous processes. Infinite-n ballooning stability analysis suggests that the configurations are mostly stable to ballooning modes (with isolated radial regions being marginally unstable). The 50 and $100 k A$ cases have a small radial region near the edge where they become ballooning unstable [27]. Overall, these equilibria all appear to be good 


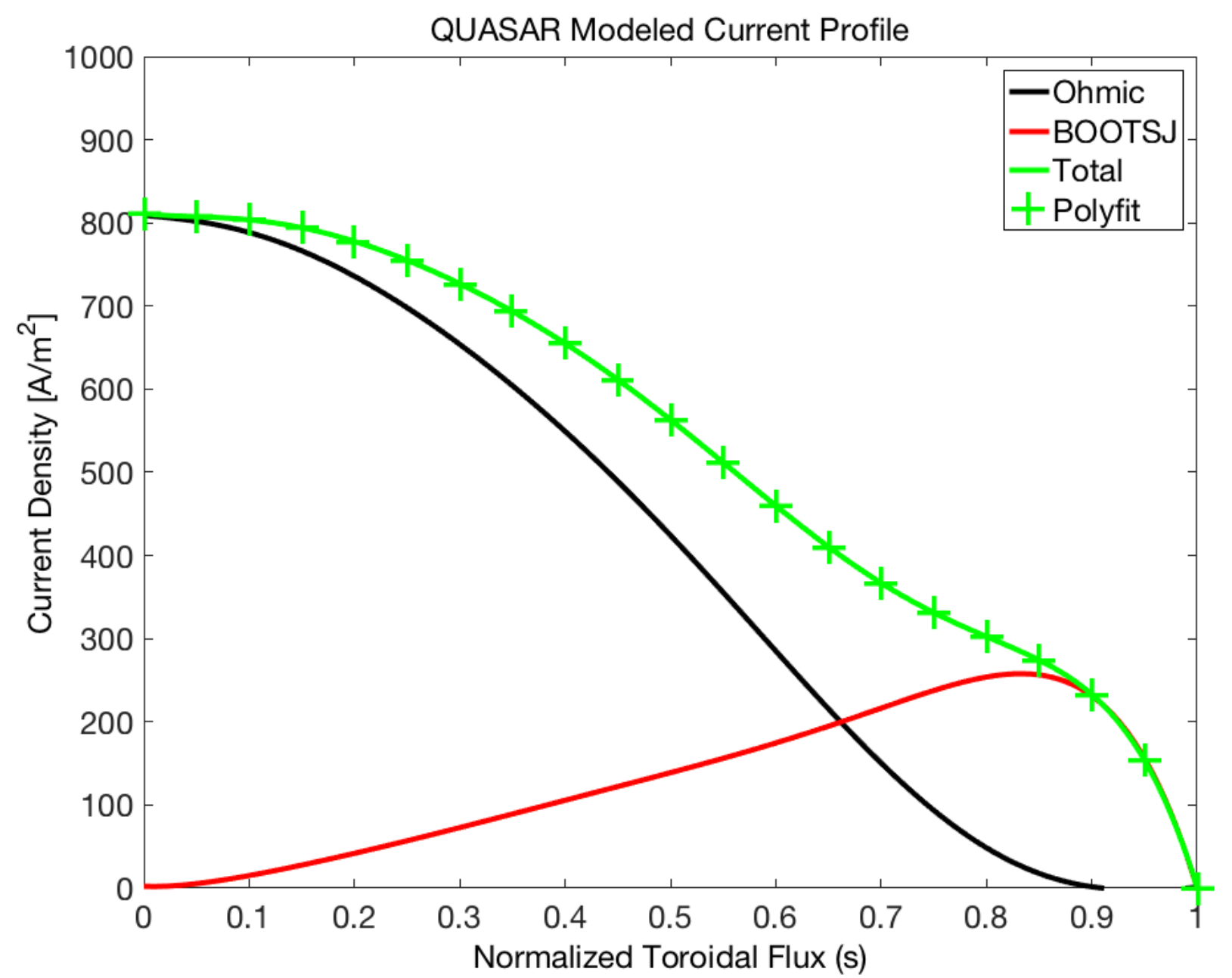

FIG. 5: Modeled bootstrap current profile (smoothed) and resulting change in current profile. Inclusion of bootstrap current would only slightly modify the rotational transform profiles.

candidates for real experimental configurations which can test the response of turbulence to magnetic shear changes.

Before moving on to the studies of turbulent transport, a digression regarding our neglect of a bootstrap current should be presented. The neglect of a bootstrap contribution to the current profile was motivated out of convenience. Namely our equilibrium code requires we specify a net toroidal current and a profile for $d I / d s$ in terms of normalized toroidal flux. For an ohmic discharge, assuming a current profile in the shape of the electron temperature is a reasonable simplification. This of course does not take the place of a full transport simulation, but such tools are still in their infancy for stellarators [28]. In addition, using configurations with relatively low plasma $\beta$ the effects of bootstrap currents are subsequently 


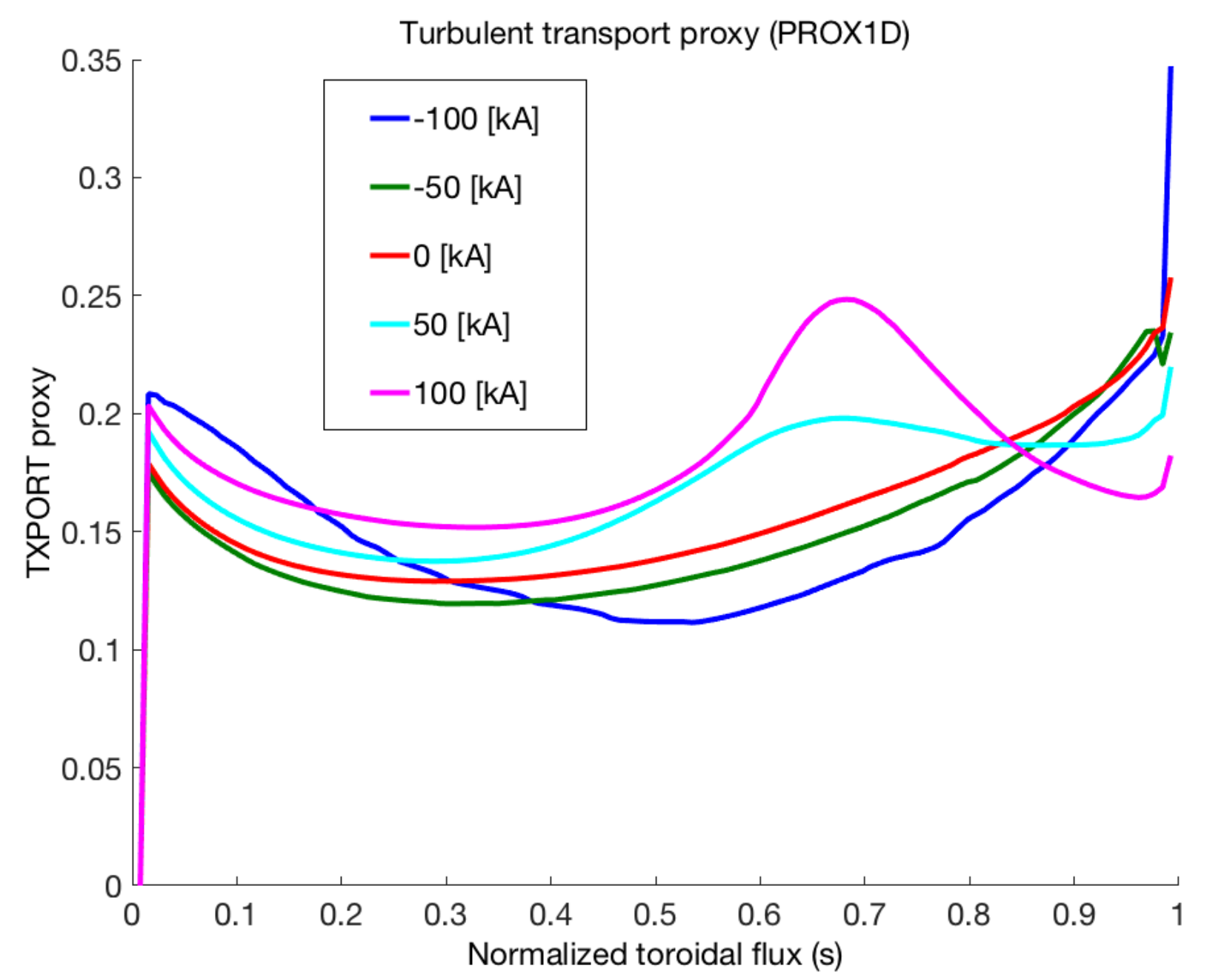

FIG. 6: Profiles of the turbulent transport proxy for the five QUASAR equilibria. A increase in the proxy is present at $s=0.7$ in the tokamak-like cases, which is consistent with ballooning calculations.

minimized. Figure 5 depicts the bootstrap current as calculated in the collisionless limit for the $0 k A$ applied toroidal field case [29]. The plotted profile has been smoothed to remove resonant features. Inclusion of a bootstrap contribution to the current profile modifies the edge current profile, which mostly changes the edge iota (a minor correctible change). If a collisional correction is taken into account, the effect of the edge bootstrap would be further reduced. This is because colisionality $\left(\nu^{*}\right)$ is inversely proportional to temperature, thus reducing the edge bootstrap contribution. Neglect of the bootstrap contribution is a justified simplifying assumption in this work.

Using the previously mentioned coupling between the optimizer and gyrokinetic tools, 
profiles of proxy and linear growth rates were evaluated. Here we've used the $Q_{p r 1 d}=<$ $\gamma_{k} g^{11}>$ (prox-1d) proxy [30], where $g^{11}$ is a radial metric tensor along the flux tube and $\gamma_{k}$ is the growth rate. Brackets $<>$ indicate averaging over flux surface and sums are taken over $k$. Figure 6 depicts the turbulent transport proxy used to generate optimized stellarator configurations based on the QUASAR fixed boundary equilibrium [5]. The more 'tokamak-like' cases show an increase in the proxy around $s=0.75$. This is also the region where these equilibria become infinite-n ballooning unstable (as calculated by the COBRA code [27]). The presence of this feature is not surprising as the proxy has a ballooning-like character. The proxy results appear inconclusive in the core region $(s<0.25)$. At larger radii the proxy function seems to indicate that as configurations become more 'tokamaklike' turbulent heat flux increases. However, care should be taken as only general trend information can be extracted from this proxy and the relative amplitude comparison made here may be very approximate.

The profile of linear growth rates provides a better comparison (figure 7). Here we see that the growth rates for modes in the core $(s<0.25)$ are reduced as the configuration becomes more 'tokamak-like.' At larger radii, this trend appears to reverse with the 'stellarator-like' configuration showing the smallest growth rate. It should be noted that the ballooning like feature is no longer present for the 'tokamak-like' configurations, and that here we are only reporting the growth rates of the most unstable modes. Recent work suggests, that proper estimation of the saturated heat flux requires an ensemble of modes to be considered [31].

Non-linear flux tube simulations were performed for a select set of flux tubes. Figure 8 (left) compares the evolution of the nonlinear heat flux for three configurations in the core $(s \sim 0.1)$. The more 'tokamak-like' configuration shows a significantly reduced heat flux and the standard deviation in the heat is much smaller as well. Figure 8 (right) performs a similar comparison between heat fluxes but in the outer plasma region $(s \sim 0.5)$. As in the core the heat flux is reduced for the more 'tokamak-like' configuration. This suggest that both the proxy and most unstable linear mode fail to recover the actual behavior of the turbulence, probably due to the disparate scales involved.

The trend where more 'tokamak-like' configurations have reduced turbulence levels is confirmed through further simulations. Figure 9 shows that at all radii the outboard mid plane flux tube has reduced turbulence levels for 'tokamak-like' configurations. This is the flux tube which passes through the region of maximum curvature in the equilibria. In 


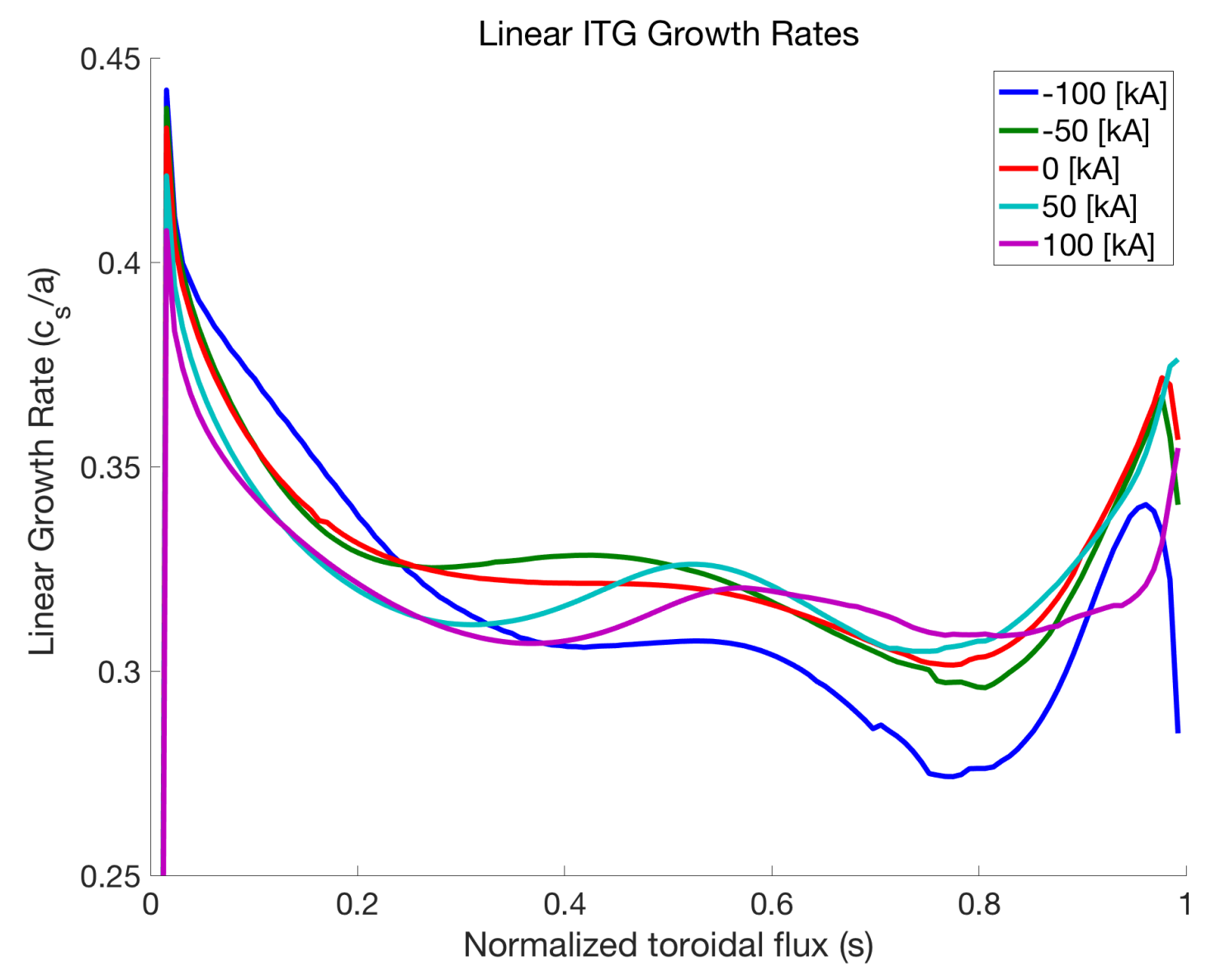

FIG. 7: Linear growth rates (most unstable Eigenmode) for ITG turbulence for the five cases of interest. The $-100 k A$ case appears to have lower growth rates in the edge region but equivalently higher growth rates in the core, as compared to the other configurations.

these plots the data points are extracted as averages of the turbulent fluctuation levels after saturation. The error bars depict the standard deviation of the turbulent heat flux after saturation. Scans of the gradient length scale suggest that the relative amplitudes of the fluxes hold. This dependency can be considered robust to profile variation. Full surface non-linear runs have also been conducted and corroborate these results, suggesting that the 'tokamak-like' configuration has lower levels of turbulent heat-flux.

\section{DISCUSSION}

In this work a series of magnetic configurations are developed which show the possibility of testing electrostatic ITG turbulence in the QUASAR device. This series of five equilibria 

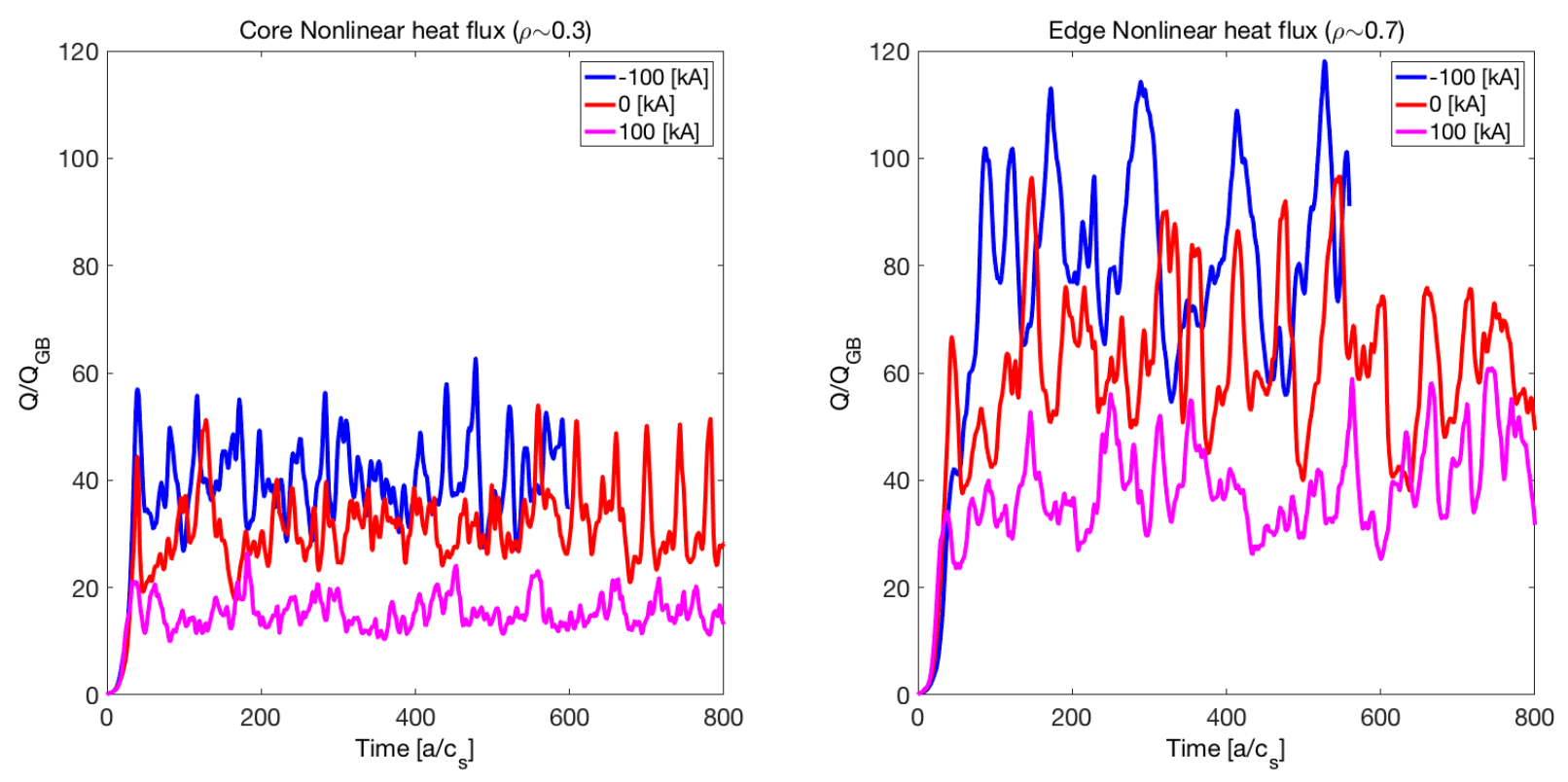

FIG. 8: Core (left) and edge (right) nonlinear heat fluxes as calculated by GENE for similar flux tubes. A consistent reduction in heat flux is present as the configuration moves from stellarator-like $(-100[k A])$ to tokamak-like rotational transform profiles $(100[k A])$.

explore the effect of magnetic shear on turbulence where the edge iota is held fixed. Profile assumptions are made which assume ohmic heating and current drive, a unique feature of the QUASAR experiment. The auxiliary toroidal field coils of the experiment play an important role in these configurations. Non-linear gyrokinetic simulations of electrostatic ITG turbulence confirm a consistent reduction in turbulent transport for configurations deemed to have a 'tokamak-like' rotational transform profile. This suggests that, should the device be built, QUASAR could play a major role in the validation of stellarator gryrokinetic theory.

In this work, an ohmic $\beta \sim 2 \%$ discharge is assumed for simplicity. A full transport simulation would provide a much more convincing basis for the study. However, in this work nearly order of magnitude changes in turbulence levels are present at all radii. This suggests that while these profile assumptions may be approximate, the response in terms of transport most likely swamps any profile discrepancies. Moreover, evaluation of the bootstrap current suggests that its contribution would be small compared to the ohmic heating profile.

Differences between proxy function, linear growth rates, and turbulent heat flux should also be addressed. The proxy functions do not claim to predict turbulent heat flux levels, but 


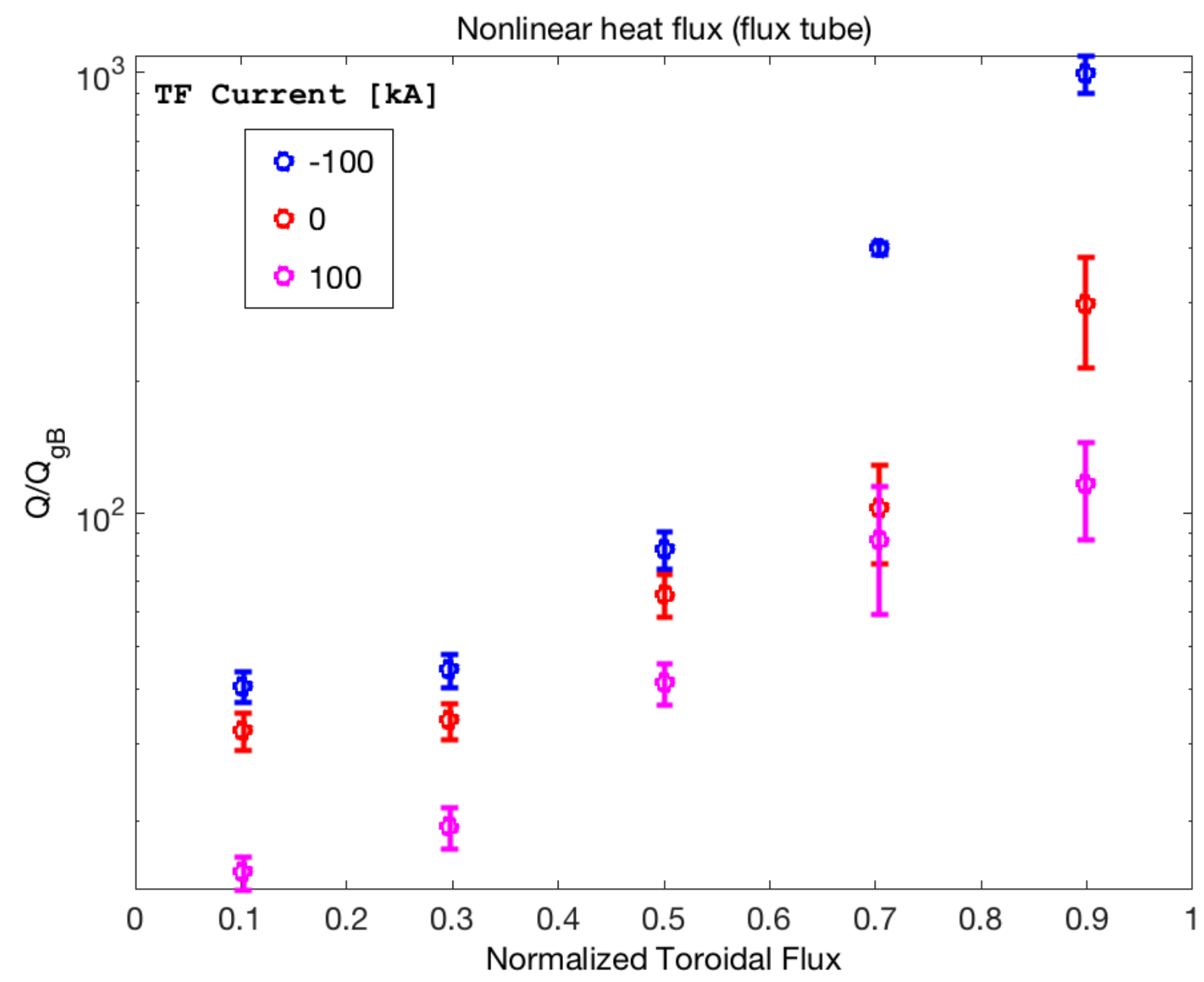

FIG. 9: A significant reduction in the turbulent heat flux all radii is determined from non-linear simulations. At each radii, similar flux tubes were analyzed.

rather mimic the dependence of turbulence on magnetic field properties. These functions become essentially normalized, and the comparison presented here is illustrative of this fact. Specifically they may provide gradient information regarding what optimization parameters reduce turbulent transport, but do not provide absolute values which allow comparison of different configurations. One may ask if the optimizer had been started from the "stellaratorlike' configuration and optimized using the proxy, would it create a configuration similar to the 'tokamak-like' case? Such work is left to future analysis. Discrepancies between the linear growth rate are indicative of missing physics in the proxy. Recent work has suggested that the saturated heat flux may depend on a combination of linear modes [31]. Our analysis focused on just the most unstable mode. 
Overall this work motivates the necessity of a device like QUASAR to better explore ITG turbulence through variation of magnetic configuration. The work also suggests that stellarators with more 'tokamak-like' rotational transform profiles will have improved transport properties. This may suggest a new field of optimization for quasi-axisymmetric stellarators. Can the stellarator bootstrap current be optimized to provide similar rotational transforms? The answers to such questions are left to future work.

\section{Acknowledgments}

Notice: This manuscript is based upon work supported by the U.S. Department of Energy, Office of Science, Office of Fusion Energy Sciences, and has been authored by Princeton University under Contract Number DE-AC02-09CH11466 with the U.S. Department of Energy. The publisher, by accepting the article for publication acknowledges, that the United States Government retains a non-exclusive, paid-up, irrevocable, world-wide license to publish or reproduce the published form of this manuscript, or allow others to do so, for United States Government purposes.

[1] H. E. Mynick, Phys. Plasmas 13, 058102 (2006).

[2] J. G. C. D. Beidler, Y. Feng, H. Maassberg, and N. B. Marushchenko, Plasma Phys. Control. Fusion 57, 014004 (2015).

[3] J. M. Canik, D. T. Anderson, F. S. B. Anderson, K. M. Likin, J. N. Talmadge, and K. Zhai, Phys. Rev. Let. 98, 085002 (2007).

[4] D. R. Mikkelsen, H. Maassberg, M. C. Zarnstorff, C. D. Beidler, W. A. Houlberg, W. Kernbichler, H. Mynick, D. A. Spong, P. Strand, and V. Tribaldos, Fusion Sci. and Tech. 51, 166180 (2007).

[5] H. E. Mynick, P. Xanthopoulos, B. Faber, M. Lucia, M. Rorvig, and J. N. Talmadge, Plasma Phys. Control. Fusion 56, 094001 (2014).

[6] P. Xanthopoulos, G. G. Plunk, A. Zocco, and P. Helander, Phys. Rev. X 6, 021033 (2016), URL https://link.aps.org/doi/10.1103/PhysRevX.6.021033.

[7] B. Coppi and F. Pegoraro, Nuclear Fusion (1977). 
[8] F. Romanelli and S. Briguglio, Physics of Fluids B: Plasma Physics 2, 754 (1990).

[9] B. Nelson, L. Berry, A. B. Brooks, M. J. Cole, J. C. Chrzanowski, H. M. Fan, P. J. Fogarty, P. L. Goranson, P. J. Heitzenroeder, S. P. Hirshman, et al., Fusion Eng. and Design 6668, 169174 (2003).

[10] M. C. Zarnstorff, L. A. Berry, A. Brooks, E. Fredrickson, G. Y. Fu, S. Hirshman, S. Hudson, L.-P. Ku, E. Lazarus, D. Mikkelsen, et al., Plasma Phys. and Control. Fusion 43, A237 (2001).

[11] D. A. Gates, A. H. Boozer, T. Brown, J. Breslau, D. Curreli, M. Landreman, S. Lazerson, J. Lore, H. E. Mynick, G. H. Neilson, et al., Nuclear Fusion 57, 126064 (2017).

[12] N. Pomphrey, A. Boozer, A. Brooks, R. Hatcher, S. P. Hirshman, S. Hudson, L.-P. Ku, E. A. Lazarus, H. Mynick, D. Monticello, et al., Fusion Sci. and Tech. 51, 181 (2007).

[13] J. T. Peterson, G. J. Hartwell, S. F. Knowlton, J. Hanson, R. F. Kelly, and C. Montgomery, Journal of Fusion Energy 26, 145 (2007), ISSN 1572-9591, URL http://dx.doi.org/10. $1007 /$ s10894-006-9053-2.

[14] T. Görler, A. E. White, D. Told, F. Jenko, C. Holland, and T. L. Rhodes, Physics of Plasmas 21, 122307 (2014).

[15] S. P. Hirshman and J. C. Whitson, Phys. Fluids 26, 3553 (1983).

[16] D. Spong, S. Hirshman, L. Berry, J. Lyon, R. Fowler, D. Strickler, M. Cole, B. Nelson, D. Williamson, A. Ware, et al., Nucl. Fusion 41, 711 (2001).

[17] S. A. Lazerson, Stellopt, URL https://www.osti.gov/doecode/biblio/12551-stellopt.

[18] F. Jenko, W. Dorland, M. Kotschenreuther, and B. N. Rogers, Physics of Plasmas 7, 1904 (2000).

[19] T. Dannert and F. Jenko, Physics of Plasmas 12, 072309 (2005).

[20] S. P. Hirshman, W. I. van Rij, and P. Merkel, Computer Physics Communications 43, 143 (1986).

[21] H. Akima, Communications of the ACM 17, 18 (1974).

[22] J. Pujol, Geophysics 72, W1 (2007).

[23] S. Lazerson and the DIII-D Team, Nuclear Fusion 55, 023009 (2015).

[24] P. Xanthopoulos, W. A. Cooper, F. Jenko, Y. Turkin, A. Runov, and J. Geiger, Physics of Plasmas 16, 082303 (2009).

[25] J. H. E. Proll, H. E. Mynick, P. Xanthopoulos, S. Lazerson, and B. J. Faber, Plasma Physics and Controlled Fusion pp. 1-9 (2015). 
[26] V. V. Nemov, S. V. Kasilov, W. Kernbichler, and M. F. Heyn, Physics of Plasmas 6, 4622 (1999).

[27] R. Sanchez, S. P. Hirshman, J. C. Whitson, and A. S. Ware, Journal of Computational Physics 161, 576 (2000).

[28] Y. Turkin, H. Maaßberg, C. D. Beidler, J. Geiger, and N. B. Marushchenko, Fusion Science and Technology 50, 387 (2006).

[29] K. C. Shaing, B. A. Carreras, N. Dominguez, V. E. Lynch, and J. S. Tolliver, Physics of Fluids B: Plasma Physics 1, 1663 (1989).

[30] H. E. Mynick, P. Xanthopoulos, B. Faber, M. Lucia, M. Rorvig, and J. N. Talmadge, Plasma Physics and Controlled Fusion 56, 094001 (2014).

[31] M. J. Pueschel, B. J. Faber, J. Citrin, C. C. Hegna, P. W. Terry, and D. R. Hatch, Physical Review Letters 116, 085001 (2016). 